\title{
Active stress as a local regulator of global size in morphogenesis
}

\author{
D. Ambrosi ${ }^{\mathrm{a}, *}$, V. Pettinati ${ }^{\mathrm{a}, \mathrm{b}}$, P. Ciarletta ${ }^{\mathrm{b}, \mathrm{c}}$ \\ a MOX-Politecnico di Milano, piazza Leonardo da Vinci 32, 20133 Milano, Italy \\ ${ }^{\mathrm{b}}$ Fondazione CEN, piazza Leonardo da Vinci 32, 20133 Milano, Italy \\ ${ }^{c}$ CNRS and Sorbonne Universités, UPMC Univ Paris 06, UMR 7190, Institut Jean le Rond d'Alembert, 4 place Jussieu case 162,75005 Paris, France
}

Received 1 August 2014, received in revised form 30 September 2014, accepted 21 November 2014, available online 18 December 2014

While a general consensus exists that the morphogenesis of living organisms has its roots in genetically encoded information, there is a big debate about the physical mechanisms that actually mediate its control. In embryo development, cells stop proliferating at homeostasis, a target state in terms of physical conditions that can represent, for instance, the shape and size of an organ. However, while control of mitosis is local, the spatial dimension of a tissue is a global information. How do single cells get aware of that at the same time? Which is their communication mechanism? While morphogen factors are demonstrated to play a key role in morphogenesis, and in particular for shape emergence, they seem unable to produce a global control on size by themselves and, conversely, many recent experiments suggest that active mechanics plays a role. Here we focus on a paradigmatic larval structure: the imaginal disc that will become the wing of the fruit fly. By a formalization of theoretical conjectures in terms of simple mathematical models, we show that inhomogeneous stress, likely dictated by morphogenetic patterns, is an admissible mechanism to convey locally the global information of organ size.

Keywords:

Morphogenesis

Elasticity

Active stress

Size control

Self-similarity

\section{Introduction}

In life science, morphogenesis is the ensemble of biological processes that lead to the emergence of an organism shape. The orchestrated process of differentiation and duplication yields organs that have a precise shape and size. An important role in this phase is held by molecules that were first hypothesized by Alan Turing. In his seminal work [1] he described how the concentration of chemical substances in a tissue evolves in time because of reaction and diffusion processes. The density patterns, dictated by instability, can be conjectured to drive the system shaping. Even if he did not precisely identify such molecules, Turing called them morphogens to convey the idea of shape generation.

A fundamental contribution to the theory of morphogens is due to Lewis Wolpert [2], who proposed the "French flag model". The central element of this model is the spatial distribution of the concentration of specific substances in the tissue: it is detected by the cells which, according to specific thresholds, trigger the transcription of distinct sets of genes. According to this theory, there is a direct correlation between the input (the concentration level) and the output (the response of the tissue): each threshold corresponds to the border of an expression territory.

* Corresponding author.

E-mail address: davide.ambrosi@polimi.it (D. Ambrosi).
Since 1970s many efforts have been made to understand how morphogens influence growth [5-9] and how cells can sense and respond to their concentration changes [10]. These studies allowed to deeper understand morphogen properties, so Turing's idea was retained, but extended: not only morphogens are responsible of pattern formation, they also specify mutual cell position and then influence organism growth [11]. The first morphogen, the bicoid, was discovered in 1988 by Christiane Nüsslein-Volhard; after that, many others have been identified: Decapentaplegic (Dpp), Hedgehog, Wing-less, Epidermal growth factor, Fibroblast growth factor and Retinoic acid, to cite a few.

While the theoretical argument that the concentration of a morphogen can drive growth is fascinating and successful in some cases, such an appealing explanation fails in many morphogenetic processes or, at least, it is to be corroborated by other physical mechanisms. The most popular model organisms in morphogenetic studies is probably the Drosophila melanogaster, also known as fruit fly. The importance of many morphogens like DPP, wingless, hedgehog and others in shaping is well assessed, because in many cases it has been observed that their absence inhibits a correct evolution of the organ. However, it is still a matter of debate how morphogens control growth in the drosophila fly and, in particular, how cells "know" when the final size has been achieved.

We concentrate our attention on the Drosophila wing imaginal disc, the structure of the larva from which the adult insect wing orig-inates. Three different regions can be identified along the proximal- 


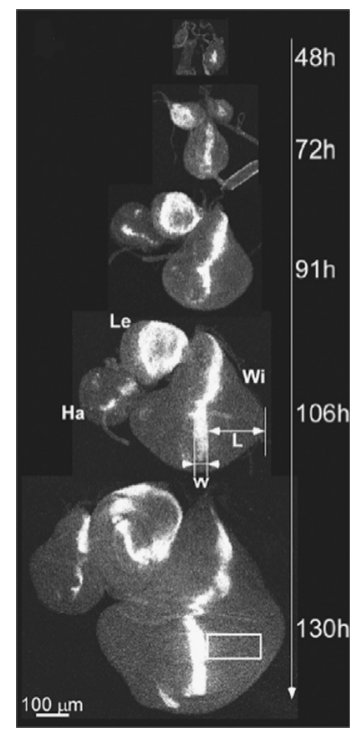

Fig. 1. Images of Dpp gradients inside wing (Wi), leg (Le), and haltere (Ha) discs at different developmental times. The parameters $w$ and $L$ represent the source and target widths, respectively; the scale bar is $100 \mu \mathrm{m}$. Reprinted with rights from [16].

distal axis of the disc: the notum (proximal), the hinge (central) and the pouch (distal). The notum will give rise to the torax, the pouch to the wing and the hinge to the flexible region between the two. The growth of the disc lasts about $150 \mathrm{~h}$ and during this morphogenetic stage the initial number of cells duplicates about 10 times, passing from 50 to 50 000 and eventually covering an area of about $1 \mathrm{~mm}^{2}$, as depicted in Fig. 1. The wing disc is a sheet of epithelial cells, a very thin structure that can be effectively represented by a two-dim-ensional approximation.

One of the most studied morphogens is the Dpp, a bone morphogenetic protein that has a key role in the development of the fruit fly, since it has been proved that the genetic suppression of its production inhibits the morphogenesis [12-14]. It spreads from a central stripe of the disc that divides it into two almost equal parts: the anterior and the posterior one. This morphogen has been widely analyzed: thanks to techniques like fluorescence recovery after photobleaching, its production rate, effective diffusion coefficient and degradation rate [15] are precisely determined. The following experimental facts are well assessed.

- The spatial dynamics of Dpp is fast with respect to the growth

of the disc: diffusion and decay of Dpp can be assumed to be always in mutual equilibrium so that the mass convection due to the material displacement caused by the cell duplication process does not distort the morphogen concentration field $c(x, t)$.

- In the early development the cell duplication rate is homogeneous in space, but changes during time: it is higher in the initial phase than in the final one. The disc area $S(t)$ evolves in time according to a Gompertzian law:

$\log (\dot{S} / S)=b_{1}-b_{2} t$

with $b_{1}$ and $b_{2}$ positive constants. At a later stage, typically after $72 \mathrm{~h}$, the mitosis is oriented along the proximal-distal axis [27,28].

In spite of the big amount of experimental data available, the res-earch about Dpp spreading dynamics is still very active: recent studies show that Dpp profile scales in space with the disc length and the morphogen concentration adapts to disc size [16-18]. Such an experi-mental behavior reported by Wartlick and coworkers [17] is explained by the authors themselves assuming that an expander of constant mass dilutes during to cell division: if the degradation rate depends linearly on it, the morphogen concentration scales as the length of the domain (expander-dilution mechanism). An alternative hypothesis which might provide scaling of the morphogen is the expansionrepression mechanism proposed by Ben-Zvi and Barkai [19]: when the disc grows, the cells at the periphery of the imaginal disc experience morphogen levels below a given threshold, and they might produce an expander that diffuses in the tissue and reduces morphogen degradation.

Notwithstanding, a full explanation of the determination of the size of an organ in terms of pure reactive-diffusing agents remains elusive. One of the motivations is that even though chemical signaling scales with length, it has a very small concentration at the boundary of the disc. This remark suggested a different approach to Hufnagel and coworkers [18]; they argue that mechanical stress might compensate the decay of morphogen concentration in the periphery of the disc, so that a combination of morphogen diffusion and mechanics might be the key regulator of disc growth. This explanation is in agreement with a number of recent reports on the emerging role of mechanics in morphogenesis [20]. Mechanical stress is known to play a role in tissue development [21], sometimes in conjunction or superposition with chemical signaling [23]. In particular, evaluation of the stress in the wing imaginal disc by photoelasticity has been the subject of recent experimental works [24,49]. Their main results are that the stress is inhomogeneous, larger in the center than in the periphery, it is compressive and grows with the size of the disc.

One of the aims of this paper is to address whether a continuum mechanics model can reproduce a stress pattern qualitatively similar to the reported one. Moreover, we are interested in investigating whether a purely mechanical setting can be provided a signaling mechanism for size regulation during growth. While the complex interaction of morphogenetic factors will not be directly addressed, a possible output of concentration pattern will be used as a datum to modulate the activation of the mechanical stress.

The tensional state in a tissue can be due to external loads, residual stress and, most notably in the present context, we argue that it is actively produced by the cells, thanks their own actomyo$\sin$ network. The stress is therefore a long range field, natural candidate for intercellular communication, and, as a matter of fact, cells are well known to modulate their motility and reproduction rate on the basis of their own tensional state [26]. Here, we formalize different conjectures about possible morphogenetic mechanisms in mathematical equations and analyze them in terms of physical admissibility. Our standpoint is that a physical field is an admissible mechanism of local transduction of global information if, under homogeneous growth, it depends in a specific functional form on the domain size and on its relative position in the organ only. Under this hypothesis, we show that a non-homogeneous stress field, possibly modulated by morphogen concentration, can be advocated as admissible mechanism of global information transmission.

This paper is organized as follows. First, we revisit, in a mathematically formal way, the most common physical mechanisms assumed in the literature for the role of the Dpp in the imaginal disc morphogenesis. We reformulate different conjec-tures in terms of mathematical models to clarify the weak and strong points of apparently opposite theories. Then, we propose a model of disc development where the cell duplication is driven by the spatial variation of mechanical tension, produced by cell contraction and spatially modulated by morphogen concentration. We argue that tissue growth is driven by an inhomogeneous mechanical stress: as its gradient drops below a threshold, the disc does not increase beyond a critical size. As discussed in the final remarks, the proposed mechanism does not suffer of 
the theoretical inconsistencies of other models, thus reconciling the roles of morphogens and mechanics.

\section{Direct control of the morphogen on growth}

Many morphogenetic factors are known to cooperate to the shaping of drosophila wing; in this section we summarize some results about the dynamics of Dpp, in order to point out the typical conceptual difficulties that are encountered when trying to account for long range ("organ-size") interactions.

While the key role of Dpp in Drosophila morphogens is widely acknowledged, its actual dynamics is controversial: according to a classical theory, it would behave as a standard macromolecule, transported by Brownian motion with flux proportional to its own local gradient. A linear diffusion coefficient $D$ plus a constant degradation rate $k$ yield to predict a constant decay length $\lambda_{0}=\sqrt{D / k}$. While cells are well known to be able to measure differences in concentrations and to regulate their duplication rate accordingly, such a simple dynamics of Dpp cannot be instructive for describing growth of an organ [29]: the Dpp slope would not change in time while the disc elongates from few to a thousand microns, and then it could not provide any local information about the size of the wing, while the concentration becomes rapidly non-appreciable far from the source. In other words, a growth rate dictated by the absolute value of Dpp with the above dynamics is not compatible with the observed homogeneous growth that stops when the wing reaches a given size.

A possible solution of this dilemma is based on the ansatz that it exists a (non-identified) "diluted" field $d(x, t)$, which is produced in the differentiation stage and then preserves its total mass constant during time: when cells duplicate, the wing surface expands and $d$ is passively diluted, so that its density decreases proportionally to the areal growth. The connection between the Dpp dynamics and the dilution of $d$ is provided by Wartlick and coworkers [17] when they observe that the decay length of the morphogen is not constant, but grows linearly with the wing size.

To recast this idea in a formal way, we consider a transverse section of the posterior part of the wing disc, where the position spans the interval $[0, L(t)]$. The morphogen source is located in $x=0$ where Dpp is produced at a constant rate $\alpha>0$, while it cannot flow at the right boundary $L(t)$. The qualitative description above rewrites in the following differential model, where $c(x, t)$ represents the Dpp concentration and, without loss of generality, we take $d(x, t=0)=1$ :

$-D \frac{\partial^{2} c}{\partial x^{2}}=-k d c$

$\left.\frac{\partial c}{\partial x}\right|_{x=0}=-\alpha,\left.\quad \frac{\partial c}{\partial x}\right|_{x=L}=0$

$L^{2} d=L_{0}^{2}$

The general solution of (1) is

$c=c_{0} \exp (-x / \lambda)+c_{1} \exp (x / \lambda)$,

where $\lambda(t)=\sqrt{D /(d(t) k)}$. Enforcing the boundary conditions gives

$c(x, t)=\alpha \lambda \frac{\cosh ((x-L) / \lambda)}{\sinh (L / \lambda)}$

In a standard scenario, $\lambda$ is constant and the concentration field (4) does not provide any information about the length of the domain $L(t)$. In particular, when $L \gg \lambda_{0}$,

$c(x, t) \simeq \alpha \lambda \exp \left(-\frac{x}{\lambda}\right)$,

the morphogen concentration decays exponentially far from the source and becomes rapidly inappreciable.

Conversely, if the "dilution" hypothesis (3) applies, the decay length scales with the domain size as

$\lambda(t)=\sqrt{D /(d(t) k)}=\lambda_{0} L(t) / L_{0}$

so that, the morphogen concentration (4), in every point of the wing, depends on $(x, t)$ in the following specific form:

$c(x, t)=L(t) \hat{c}(x / L(t))$.

During the homogeneous expansion each material point (and therefore every cell) preserves its relative position $x / L(t)$ and, according to the solution (6), gets an immediate information on the wing size by direct measure of the local concentration. Notice that the value of $c$ itself is a measure of the domain size, without need to invoke further spatial or time derivatives. The numerical solution of the system of Eqs. (1)-(3) is plotted versus experimental data in Fig. 2.

\section{Growth controlled by mechanical factors}

The authors of the "scaling decay length" theory support their arguments by experiments; however the issue remains controversial. Cells in which the increase of Dpp signaling levels was genetically
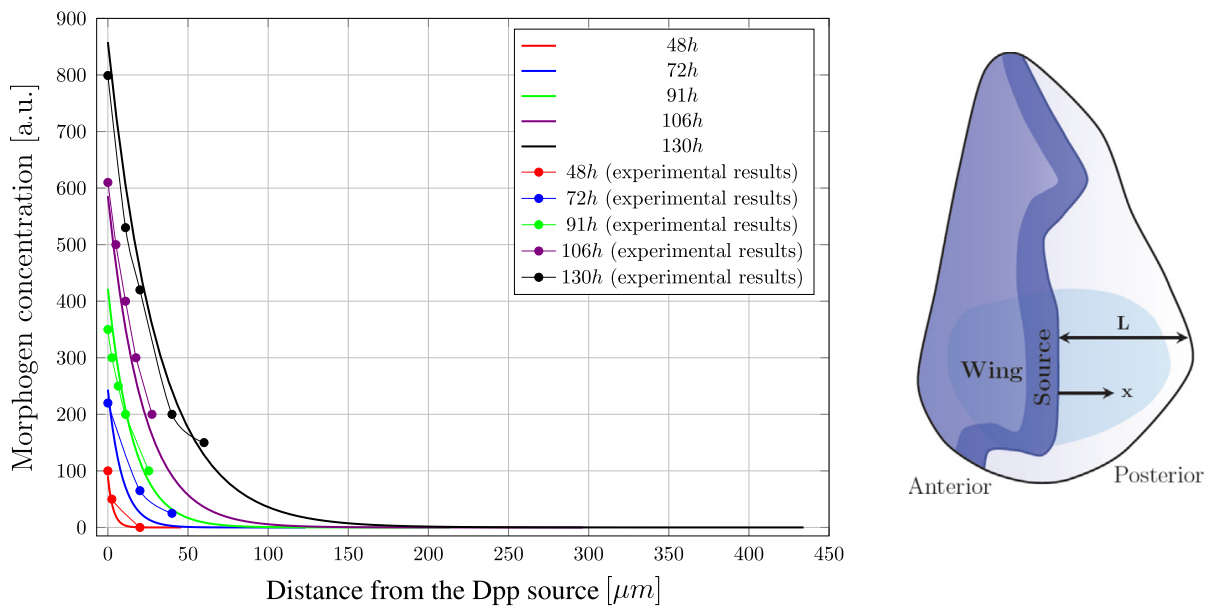

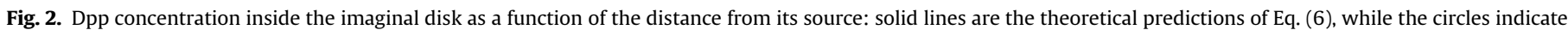
the experimental data, taken from [16]. The material parameters are collected in Table 1. 
abrogated grew at rates comparable to those of wild-type cells [30]. Moreover no diluted factor has been identified yet and a gradient of Dpp is not necessary for cell proliferation $[31,32,13]$.

An alternative approach to explain how the size in the imaginal disc growth is controlled by a limited proliferation is based on mechanobiology [18]. Cells are well known not only to produce force but also to modulate their behavior (for instance in polarization, migration, patterning and duplication) depending on the stiffness of the environment and on the externally applied load, possibly interacting with the one they actively produce [33]. Stress is therefore a natural candidate to transmit information on the size of the domain. However, a classical load-stress mechanical theory would be useless for the present purposes: since the growth time scale is very long with respect to the relaxation times of the cellular material, viscosity plays no role, the tensional state in the wing depends on the strain only and it is equal to the load (if any) on the boundary, whatever its rheological properties are.

The scenario dramatically changes if the active stress produced by the cells is introduced in the force balance. It is well known that cells have the ability to actively produce forces: they are polarized in the embryo and the cortical actomyosin machinery orientated along the radial direction produces a hoop stress [34]. In our prototypical system, the imaginal disc of the Drosophila wing, cells are apparently polarized in a circumferential fashion [35]. We are therefore interested in investigating whether a direct relation between circumferential tension and domain size can be compatible with the nature of mechanical stress; following the same approach of the previous section, we discuss whether stress can provide a local measure of a global information as the domain size.

In the following we assume radial symmetry of the problem: a characterization suggested by the discoidal shape of the wing pouch and by the pattern arising from the combined action of different morphogens. For several authors [18,50,51] cell proliferation in the wing pouch is stimulated by a central point source, at the intersection of line sources of DPP and Wingless; moreover, Wingless is expressed in two concentric rings around the wing pouch [52]. However, we remark that the actual dynamics of each morphogen and the complex cooperation among different ones is immaterial for the discussion that follows. The radial symmetry we are assuming is therefore a starting point to address the main question about a global signaling mechanism that accounts for a spatial homogeneous tissue growth and is compatible with a non-uniform distribution of local signals, like single morphogen concentrations.

\subsection{Inert matter mechanics: load at the boundary}

Since we are interested in modeling morphological changes developing in the course of several hours, we restrict our analysis to equilibrium conditions. In living matter the residual stress, which is by definition the tension existing in an unloaded body, can have two sources. It can be generated by either inhomogeneous growth or the active contraction of the cellular matter. The former is neglected in the following on the basis of the time scales of interest, assuming that it is quickly relaxed by viscous dissipation. Conversely, the force per unit surface actively produced by the cells plays a key a role in embryo development, taking values of hundreds and thousands of Pascals.

As a starting point, in this section the strain, defined with respect to the current (grown) relaxed state, is supposed to be small. This assumption yields a linear elastic model, where we emphasize that the "small strain assumption" that will be relaxed in the next section applies with respect to the imaginal disc configuration at current time.

The shape of the domain [37] suggests to enforce the radial symmetry of the problem. Let the size of the disc in its relaxed configuration be $R_{0}$ so that the radial coordinate $R \in\left[0, R_{0}\right]$ and only radial displacement $u=u(R)$ occurs. Under hypothesis of plane stress, the diagonal Cauchy stress tensor has components

$$
T_{R R}=\frac{E}{1-\nu^{2}} \frac{d u}{d R}+\frac{E \nu}{1-\nu^{2}} \frac{u}{R}, \quad T_{\Theta \Theta}=\frac{E}{1-\nu^{2}} \frac{u}{R}+\frac{E \nu}{1-\nu^{2}} \frac{d u}{d R},
$$

where $E$ and $\nu$ are the Young modulus and the Poisson ratio, respectively. According to [25], we set $E=100 \mathrm{kPa}$ and $\nu=0.5$, to account for incompressibility. The stress balance equation

$\frac{d T_{R R}}{d R}+\frac{1}{R}\left(T_{R R}-T_{\Theta \Theta}\right)=0$

rewrites

$u^{\prime \prime}+\frac{1}{R}\left(u^{\prime}-\frac{u}{R}\right)=0$,

where the prime denotes differentiation with respect to $R$. Imposing the boundary conditions

$\left.u\right|_{0}=0,\left.\quad T_{R R}\right|_{R_{0}}=\sigma$,

we get the solution

$u(R)=\sigma \frac{1-\nu}{E} R$,

which yields radial and hoop stresses independent on the domain size. As expected, a simple stress field generated by a load at the boundary cannot be an effective (indirect) measure of the wing size in an "inert-matter" context.

\subsection{Surface tension}

Surface tension is an alternative physical mechanism that might provide a tensional status in the imaginal disc depending on its own size. It classically occurs at the interface between two fluids, but it has also been introduced to account for the ability of cell aggregates to preserve their spherical shape [38]. The balance equation (8) with boundary condition

$$
\sigma=-\frac{\sigma_{0}}{R_{0}}
$$

where $\sigma_{0}$ is the surface tension coefficient and it has solution

$u(R)=-\sigma_{0} \frac{1-\nu}{E} \frac{R}{R_{0}}$,

and then

$T_{R R}=T_{\Theta \Theta}=-\frac{\sigma_{0}}{R_{0}}$.

This explanation is very attractive for its simplicity. Unfortunately a simple surface tension has magnitude much smaller than the internal forces that originate the stress state in a layer of epithelial cells: external loads have no relevance, while the main player is the active force produced by the cells themselves. Despite some attempts in this direction [39], the internal active forces per unit surface have a volumetric nature and they are not in general equivalent (they cannot be represented as) a simple surface tension. The stress pattern actively

Table 1

Material parameters characterizing the morphogenesis of the wing imaginal disk.

\begin{tabular}{lll}
\hline Symbol & Refs. & Value \\
\hline$b_{1}$ & {$[17]$} & $7.1 \times 10^{-3}$ \\
$b_{2}$ & {$[17]$} & $6.6 \times 10^{-1}$ \\
$D$ & {$[15]$} & $3.6 \times 10^{2}\left[\mu \mathrm{m}^{2} / \mathrm{h}\right]$ \\
$k_{d}^{\mathrm{a}}$ & {$[15,16]$} & $320(t=30 \mathrm{~h})-0.4(t=130 \mathrm{~h})\left[\mathrm{h}^{-1}\right]$ \\
$\lambda^{\mathrm{a}}$ & {$[15,16]$} & $1.2(t=30 \mathrm{~h})-31.5(t=130 \mathrm{~h})[\mu \mathrm{m}]$ \\
$\sigma_{0}$ & {$[38]$} & $1-22 \times 10^{-3} \mathrm{~N} / \mathrm{m}$ \\
$E$ & {$[25]$} & $100 \mathrm{kPa}$ \\
$\nu$ & & 0.5 \\
\hline
\end{tabular}

${ }^{\text {a }}$ Calculated from the observed data. 
produced by living matter can be quite more complex, as argued in the next section.

\section{Active stress}

Despite its appealing simplicity, the idea that embryos can be mechanically described as fluids with interfacial tension has been disregarded in recent years in the light of a number of experiments that elucidate the real nature of the cell-cell interaction in cell assemblies [34]. The force per unit surface in a cellular aggregate is not a simple hydrostatic pressure, it is spatially inhomogeneous and it is produced by the interplay between cell-cell adhesion and their cortical acto-myosin cables that contract according to specific directions. The perimetral anisotropic contraction is known to promote cell migration by intercalation mechanism; here we are interested in understanding if the stress produced in such a context might also work as an intracellular signal to convey information about the domain size.

We introduce the cellular active stress assuming that Dpp or some other protein, like Dachs (which seems to depend on Dpp at some extent $[9,40])$, convey contraction in a circumferential direction. Mor-phogens have here a permissive role in polarizing the cells that pro-duce a cortical tension according to a specific orientation: apparently in a circumferential fashion here, while a radial orientation has been observed in embryos [34]. The stress is known to be concentrated in the central region of the disc; laser ablation techniques [4] allow to release the active tension produced by actomyosin cables, which has been measured to be 2.5 times larger in the anteroposterior compartment versus the rest of the tissue [3]. For the sake of simplicity we first solve the force balance equation in the context of linear elasticity (Section 5), then we extend the results to the non-linear theory (Section 6).

The force per unit surface actively produced by the cells will be mathematically encoded in an active strain formulation, formally corresponding to decompose the tensor gradient of deformation into two contributions: the passive (elastic, energy storing) one $F_{e}$ and the active one $F_{a}$, so that $F=F_{e} F_{a}$ [36]. The resulting mathematical formalism is fully analogous to the one originally proposed by Kroner and Lee in plasticity and later developed by several authors to account for the emergence of residual stress in growing soft living tissues $[42,44,45]$. This is not surprising because, by definition, residual stress is the stress that persists in an unloaded body and, in living systems, it can be produced to two possible sources: inhomogeneous growth or active contraction of the elementary constituents of the tissue itself, i.e. the cells. The former contribution is passive by nature and accounts for incompatibility of the deformation generated by the growth, while the latter is intrinsically active, it also introduces incompatibility in the relaxed configuration which vanish under a possible inhibition of the contractile activity of the cells. The application of the multiplicative decomposition framework to the active stress generation is less frequent, but not new at a tissue level [22]. Following the experimental literature, we assume that growth is homogeneous, it does not produce residual stress and it is slow enough that there is no feedback on mechanics. Intriguingly enough, possible residual stress of passive type could be however incorporated in the theory for free. As the tissue is composed by the same kind of cells that do not exhibit anisotropy in the passive regime, we place the theory in the setting of homogeneous isotropic materials: anisotropy is then provided by the constitutive assumptions for $F_{a}$.

In a step by step approach, we start merging the active strain decomposition into a plane-strain linear elasticity setting; this corresponds to assume that small elastic deformations occur with respect to the possible finite distortion of the stress-free configuration. The relaxed state is to be understood as the placement of the imaginal disc grown when no active force is triggered. The contraction of the cortical filaments is encoded in the $F_{a}$ factor of the multiplicative decomposition of the gradient of deformation tensor $F$, representing the strain of the microstructure [41]. The linear setting is of limited applicability but provides results of transparent simplicity that will be generalized in the next section to the non-linear theory.

\section{Linear elasticity}

\subsection{Homogeneous active stress}

Let us consider a material point of an elastic material that, under the action of some mechanical solicitation, undergoes the displacement $\mathbf{u}=\mathbf{X}-\mathbf{X}$ from the reference (relaxed) configuration. We define the tensor gradient of deformation $F=\partial \mathbf{x} / \partial \mathbf{X}$ and we introduce a multiplicative decomposition of the tensor gradient of deformation $\mathrm{F}$ :

$\mathrm{F}=\mathrm{F}_{e} \mathrm{~F}_{a}$,

where $F_{e}$ and $F_{a}$ the elastic and the active components of $F$ respectively, and $F_{a}$ has to be constitutively prescribed. It is worth mentioning that behind this multiplicative decomposition, severe restrictions exist for the constitutive assumptions in order to enforce stability and uniqueness of the solution (see [46] for a review).

Under the same symmetry assumptions exploited in the section above, the tensor gradient of deformation depends on the radial displacement only as

$\mathrm{F}=\left[\begin{array}{cc}1+u^{\prime}(R) & 0 \\ 0 & 1+u(R) / R\end{array}\right]$,

where $u(R)=r(R)-R$ and prime denotes differentiation with respect to $R$. The radial active strain takes the form

$\mathrm{F}_{a}=\left[\begin{array}{cc}1 & 0 \\ 0 & 1 / a^{2}\end{array}\right]$

where $a>1$ accounts for the circumferential contraction. The radial and hoop components of the stress depend linearly on $\mathrm{F}_{e}=\mathrm{FF}_{a}^{-1}$ and read

$T_{R R}=\frac{E}{1-\nu^{2}} u^{\prime}+\frac{E \nu}{1-\nu^{2}}\left(\left(\frac{u}{R}+1\right) a^{2}-1\right)$,

$T_{\Theta \Theta}=\frac{E}{1-\nu^{2}}\left(\left(\frac{u}{R}+1\right) a^{2}-1\right)+\frac{E \nu}{1-\nu^{2}} u^{\prime}$

and the force balance equation (7) rewrites

$\frac{E}{1-\nu^{2}} R u^{\prime \prime}+\frac{E\left(1-\nu+\nu a^{2}\right)}{1-\nu^{2}} u^{\prime}-a^{2} \frac{E}{1-\nu^{2}} \frac{u}{R}+\frac{E}{1+\nu}\left(1-a^{2}\right)=0$.

We now look for a solution of the form

$u(R)=A R^{\beta}-R$,

where $A, \beta$ are constants.

Plugging the form (18) into (17), we find a second order algebraic equation in $\beta$ which has two roots: one positive and one negative. We discard the negative root so that the boundary condition $u(0)=0$ is fulfilled and, after observing that the positive root $\beta>1, A$ has to be fixed using the boundary condition

$T_{R R}\left(R_{0}\right)=0$.

We obtain

$u(R)=\frac{1+\nu}{\beta+\nu a^{2}} \frac{R^{\beta}}{R_{0}^{\beta-1}}-R$

We notice that this exact solution allows us to state in a precise way the admissibility condition for the applicability of a linearized theory: the strain is small if

$\frac{1+\nu}{\beta+\nu a^{2}} \beta \ll 2$. 


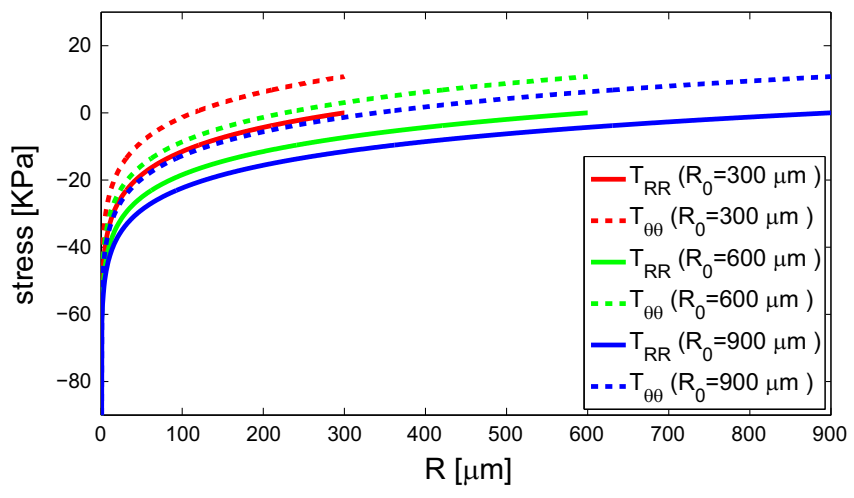

Fig. 3. Plot of the radial and circumferential stress components versus the radial coordinate $R$ at different domain sizes for a linear elastic material homogeneously stretched by the active strain (function (19)). The material parameters are $1 / a=0.9$ and $E=100 \mathrm{kPa}$ (from [25]). The radial stress is always compressive, while the hoop stress changes sign at about $R_{0} / 2$.

The radial and hoop stress then take the following form:

$\left.T_{R R}=\frac{E}{1-\nu}\left(\frac{R}{R_{0}}\right)^{\beta-1}-1\right)$
$\left.T_{\Theta \Theta}=\frac{E}{1-\nu} \frac{a^{2}+\nu \beta}{\beta+\nu a^{2}}\left(\frac{R}{R_{0}}\right)^{\beta-1}-1\right)$

Both components of the stress are plotted versus the radial coor-dinate in Fig. 3. The predicted stress has a number of interesting features: the radial component is always in compression and duly goes to zero at the boundary; the hoop stress is compressive too, its value being larger at the center than at the periphery of the wing, in agreement with the observations [24]. According to the solution (19), the stress scales with the relative position $R / R_{0}$, so that a material point is always subject to the same tensional state, whatever $R_{0}$. While the stress itself cannot be an effective size signal, its gradient does, as it is a function of size and space of the type $f\left(R / R_{0}\right) / R_{0}, f$ being constant for a material point. Intriguingly, the solution stress (19) also predicts the observed growth of the (magnitude of the) stress with size in the central region of the disc, as reported by Nienhaus et al. [24]: thanks to self-similarity of the solution, for a fixed in space radial position the pressure grows with the disc radius.

The active stress provides an effective mechanism for local transmission of global information on size and predicts a tensile pattern that is in agreement with the (few) experimental information that we have on this biophysical system. It remains however an open question if and how soluble morphogens and stress might cooperate in size determination. In the next section we introduce a possible role for morphogen concentration, that is expected to be at least permissive in morphogenesis.

\subsection{Inhomogeneous active tension}

Attempts to face the complexity of morphogenesis by mechanical arguments or reaction-diffusion dynamics only do not seem able to predict the most recent experimental observations. We therefore conjecture that the morphogen has a triggering role in activating the cells contraction in circumferential direction. This means that active hoop stress is expected to be produced only in a radius of few decay lengths from the center line.

Say $R_{i}$ the (possibly moving) distance from the center of the imaginal disc where the morphogen concentration is not any more detectable by the cells. Then Eq. (17) holds in $\left(0, R_{i}\right)$ while Eq. (8) applies in $\left(R_{i}, R_{0}\right)$. Standard boundary conditions are then to be supplemented by interface conditions in $R_{i}$ : continuity of displacement and stress.

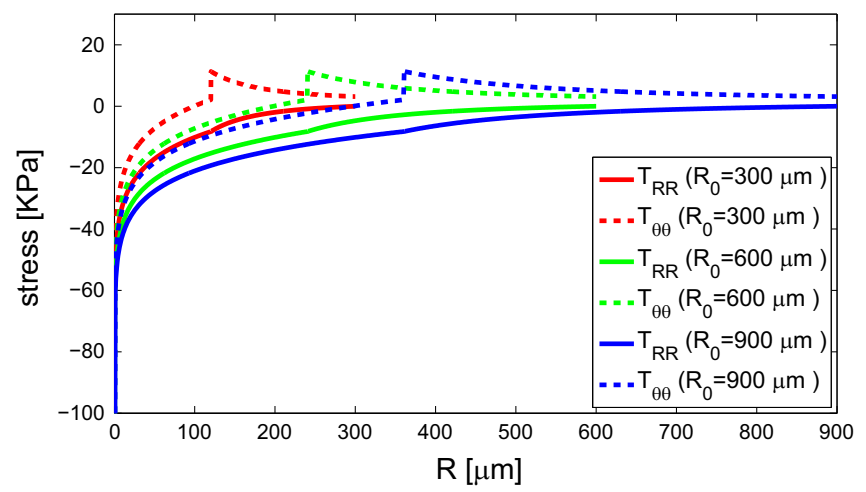

Fig. 4. Radial and hoop stress plotted versus the radial coordinate for different domain size, for a wing imaginal disk that behaves a linear elastic material where cells contract circumferentially in the center only. The functions (22) are plotted for $1 / a=0.9$, and $E=100 \mathrm{kPa}$ (from [25]). As expected, the radial stress is continuous and compressive everywhere, the hoop stress jumps at the interface and changes sign (from compressive to tensional).

The solution takes the form

$\begin{cases}u=A R^{\beta}-R, & R \in\left(0, R_{i}\right) \\ u=B R+C / R, & R \in\left(R_{i}, R_{0}\right)\end{cases}$

where $A, B, C$ are to be fixed exploiting the interface and boundary conditions

$\llbracket u \rrbracket_{R_{i}}=\llbracket T_{R R} \rrbracket_{R_{i}}=0,\left.\quad T_{R R}\right|_{R_{0}}=0$,

where $\llbracket \cdot \rrbracket_{R_{i}}$ denotes the jump of the argument at $R=R_{i}$.

Some calculations (see Appendix) yield the following form of the stress components:

$T_{R R}=\frac{E}{1-\nu}\left(\left(\frac{R}{R_{i}}\right)^{\beta-1} g_{R}\left(R_{i} / R_{0}\right)-1\right)$,
$T_{\Theta \Theta}=\frac{E}{1-\nu}\left(\left(\frac{R}{R_{i}}\right)^{\beta-1} g_{\Theta}\left(R_{i} / R_{0}\right)-1\right)$,

in $\left(0, R_{i}\right)$, where $g_{R}\left(R_{i} / R_{0}\right)$ and $g_{\Theta}\left(R_{i} / R_{0}\right)$ are explicitly determined. The radial and hoop stress are plotted in Fig. 4. Both components of the stress are now functions of the (time-dependent) domain length $R_{0}(t)$ and of the morphogen action length $R_{i}$. If the morphogen pattern scales with the domain length [17], the ratio between $R_{i}(t)$

and $R_{0}(t)$ is constant, so that $g_{R}$ and $g_{\Theta}$ are also constant. Therefore, also when there is an active tension triggered only in a portion of the imaginal disc that scales with the disc size, all the experimental evidences captured by the homogeneous model of the section above are recovered: a material cell that during the homogeneous growth process has constant relative position $R / R_{0}$ is subject to the constant stress in time, its spatial gradient is damped as $1 / R_{0}$ and it is therefore a plausible candidate as a size measure. As mitosis is well known to depend on the mechanical tensional state [43], it is perfectly plausible that cells are individually programmed to stop their duplication below a threshold tension gradient, thus producing collectively an organ of prescribed size.

Both components of the stress are compressive in the central area of the disc and, most remarkably, they grow (in magnitude) versus time for a fixed $R$, an experimentally reported feature. In fact, the absolute value of the hoop stress predicted by Eq. (22) is a decreasing function of the spatial position; as the wing size grows, the solution accommodates in a self-similar way and, for a fixed spatial coordinate $R$, the magnitude of the hoop stress grows, in agreement with experimental reports. 


\section{Non-linear elasticity}

The theory illustrated in the section above can be generalized to a fully non-linear framework assuming that the imaginal disc is made of an incompressible neo-Hookean material that undergoes an inhomogeneous active deformation. The strain energy density function is

$\Psi\left(\mathrm{F}_{e}\right)=\frac{\mu}{2} \operatorname{tr}\left(\mathrm{B}_{e}-3\right)$,

where $\mu$ is the shear modulus and $\mathrm{B}_{e}=\mathrm{F}_{e} \mathrm{~F}_{e}^{T}$. First variation of the strain energy density enforced by the incompressibility constrain det $\mathrm{F}_{e}=1$ leads to the Cauchy stress tensor:

$\mathrm{T}=\mu \mathrm{B}_{e}-p \mathrm{I}$

where $p$ is the Lagrange multiplier enforcing the incompressibility constraint.

\subsection{The 2-D axial symmetric case: homogeneous contraction}

We state the theory in a two-dimensional setting, on the basis of an assumption of small strain and stress in the axial direction. This conjecture will be discussed in more detail in final remarks. In a two-dimensional non-linear framework we assume that the decomposition (13) takes the generic form

$\mathrm{F}_{a}=\left[\begin{array}{ll}\gamma & 0 \\ 0 & \delta\end{array}\right]$

in $\left(0, R_{i}\right)$, while $\mathrm{F}_{a}$ is the identity tensor elsewhere. Specific forms of $F_{a}$ as in (14) will be recovered as particular cases. In cylindrical axial-symmetric coordinates, the deformation gradient tensor reads

$\mathrm{F}=\left[\begin{array}{cc}\frac{d r}{d R} & 0 \\ 0 & \frac{r}{R}\end{array}\right], \quad \mathrm{F}_{e}=\left[\begin{array}{cc}\frac{1}{\gamma} \frac{d r}{d R} & 0 \\ 0 & \frac{1}{\delta} \frac{r}{R}\end{array}\right]$

The incompressibility constraint

$\frac{1}{\gamma \delta} \frac{d r}{d R} \frac{r}{R}=1$

can be immediately integrated using the boundary condition $r(0)=0$

$r=\sqrt{\gamma \delta} R$

so that

$\mathrm{F}_{e}=\left[\begin{array}{cc}\sqrt{\frac{\delta}{\gamma}} & 0 \\ 0 & \sqrt{\frac{\gamma}{\delta}}\end{array}\right]$

Direct integration of the equilibrium equation

$\frac{d}{d r} T_{r r}=\frac{1}{r}\left(T_{\theta \theta}-T_{r r}\right)$

leads to the determination of the radial component of the stress:

$T_{r r}(r)-T_{r r}\left(r_{i}\right)=\mu\left(\frac{\gamma}{\delta}-\frac{\delta}{\gamma}\right) \int_{r_{i}}^{r} \frac{1}{s} d s$.

Eq. (24) implies that

$T_{r r}(R)-T_{r r}\left(R_{i}\right)=\mu\left(\frac{\gamma}{\delta}-\frac{\delta}{\gamma}\right) \int_{\sqrt{\gamma \delta} R_{i}}^{\sqrt{\gamma \delta} R} \frac{1}{S} d S$,

therefore

$T_{r r}=T_{r r}\left(R_{i}\right)+\mu\left(\frac{\gamma}{\delta}-\frac{\delta}{\gamma}\right) \log \left(\frac{R}{R_{i}}\right)$.

The expression of the radial stress allows us to calculate the pressure $p$ and then obtain the hoop stress:

$T_{\theta \theta}=\mu\left(\frac{\gamma}{\delta}-\frac{\delta}{\gamma}\right)\left(1+\log \left(\frac{R}{R_{i}}\right)\right)+T_{r r}\left(R_{i}\right)$

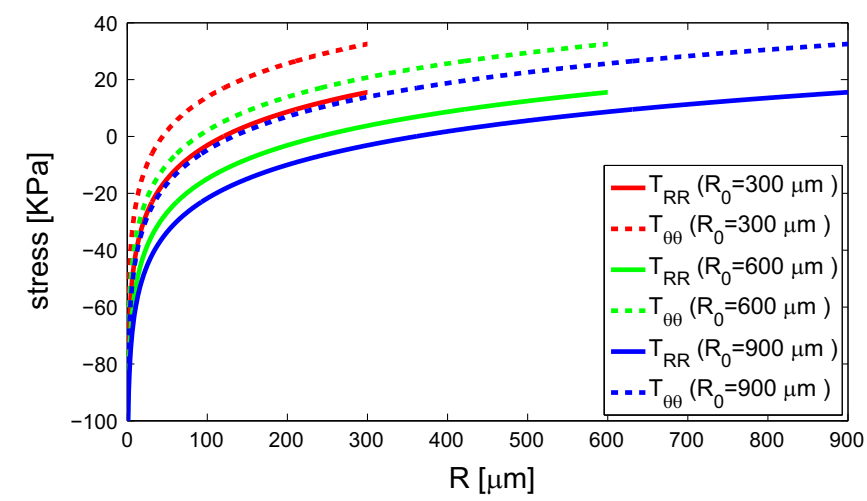

Fig. 5. Plot of the radial and circumferential stress components versus the radial coordinate $R$ at different domain sizes for a non-linear elastic material. Here the growth occurs overall the domain, $\mu=40$ and $\gamma=1$ and $\delta=(1 / 0.9)^{2}$. Not surpris-ingly, the functions strongly resemble the results of the linear theory.

Both the radial and the circumferential components of the stress exhibit a singularity at the origin, unless $\gamma=\delta$; in such a case, the stress is constant in $\left(0, R_{i}\right)$. If the contraction is activated in the whole disc, $R_{i}=R_{0}$ and the stress has the form in (25) and (26), where the contribution in $R_{i}$ vanishes. The functions (25) and (26) are plotted in Fig. 5.

If $\gamma>\delta(\gamma<\delta)$ the cells undergo a tension (compression) both in the radial and in the circumferential direction. As in the linear case, the stress magnitude decreases towards the periphery and grows with the disc dimension in an absolute position $R$. In analogy, with the linear homogeneous case of Section 5.1, during the developmental process each material point is subject to the same level of stress, but, in contrast with the linear approximation, also the stress gradient does not scale with size. In the next section we show how the introduction of an inhomogeneous contractility controlled by a scaling morphogenetic factor resolves this difficulty.

\subsection{Inhomogeneous contraction pattern}

If the radius of the mechanically active disc $R_{i}$ is smaller than $R_{0}$, standard (purely passive) elasticity equations are to be solved in $\left(R_{i}, R_{0}\right)$ in order to provide the interface radial component of the stress $T_{r r}\left(R_{i}\right)$. Incompressibility is here expressed by the following equation:

$\frac{r}{R} \frac{d r}{d R}=1$,

that can be integrated to give

$r(R)=\sqrt{R^{2}-R_{i}^{2}+r_{i}^{2}}$.

The solution of the equilibrium equation yields the determination of the radial stress:

$T_{r r}\left(r_{0}\right)-T_{r r}(r)=\int_{r}^{r_{0}} \frac{\mu}{s}\left(\frac{s^{2}}{s^{2}-\kappa R_{i}^{2}}-\frac{s^{2}-\kappa R_{i}^{2}}{s^{2}}\right) d s$

where $r_{i}^{2}-R_{i}^{2}=(\gamma \delta-1) R_{i}^{2}=\kappa R_{i}^{2}$. The integral in (28) can be conveniently calculated after a change of variables:

$T_{r r}\left(R_{0}\right)-T_{r r}(R)=\int_{\sqrt{R^{2}+\kappa R_{i}^{2}}}^{\sqrt{R_{0}^{2}+\kappa R_{i}^{2}}} \frac{\mu S}{S^{2}+\kappa R_{i}^{2}}\left(\frac{S^{2}+\kappa R_{i}^{2}}{S^{2}}-\frac{S^{2}}{S^{2}+\kappa R_{i}^{2}}\right) d S$.

The boundary condition $T_{r r}\left(R_{0}\right)=0$ allows the determination of $T_{r r}$ and $T_{\theta \theta}$ in $\left(R_{i}, R_{0}\right)$ :

$\left.T_{r r}=\frac{\mu}{2}\left[\log \frac{\left(R_{0}^{2}+2 \kappa R_{i}^{2}\right)\left(R^{2}+\kappa R_{i}^{2}\right)}{\left(R^{2}+2 \kappa R_{i}^{2}\right)\left(R_{0}^{2}+\kappa R_{i}^{2}\right)}\right)+\kappa R_{i}^{2} \frac{R^{2}-R_{0}^{2}}{\left(R_{0}^{2}+2 \kappa R_{i}^{2}\right)\left(R^{2}+2 \kappa R_{i}^{2}\right)}\right]$ 


$$
\begin{aligned}
T_{\theta \theta}= & \mu\left[\frac{R^{2}+\kappa R_{i}^{2}}{R^{2}}-\frac{R^{2}}{R^{2}+\kappa R_{i}^{2}}+\frac{\kappa}{2} R_{i}^{2} \frac{R^{2}-R_{0}^{2}}{\left(R_{0}^{2}+2 \kappa R_{i}^{2}\right)\left(R^{2}+2 \kappa R_{i}^{2}\right)}\right. \\
& \left.+\frac{1}{2} \log \left(1+\frac{\kappa R_{i}^{2}\left(R^{2}-R_{0}^{2}\right)}{\left(R^{2}+2 \kappa R_{i}^{2}\right)\left(R_{0}^{2}+\kappa R_{i}^{2}\right)}\right)\right]
\end{aligned}
$$

and the (unique) value of the radial stress in $R_{i}$ can be evaluated: $T_{r r}\left(R_{i}\right)=\frac{\mu}{2}\left[\log \left(\frac{\left(R_{0}^{2}+2 \kappa R_{i}^{2}\right)\left(R_{i}^{2}+\kappa R_{i}^{2}\right)}{\left(R_{i}^{2}+2 \kappa R_{i}^{2}\right)\left(R_{0}^{2}+\kappa R_{i}^{2}\right)}\right)+\kappa R_{i}^{2} \frac{R_{i}^{2}-R_{0}^{2}}{\left(R_{0}^{2}+2 \kappa R_{i}^{2}\right)\left(R_{i}^{2}+2 \kappa R_{i}^{2}\right)}\right]$ so that also the solution in the inner region (25) and (26) is completely determined. The stress components are plotted in Fig. 6 at growing disc size, and in Fig. 7 for different magnitude of active contraction.

The stress field (29) and (30) has the due scaling properties for a scaling morphogenetic pattern.

\section{Final remarks}

According to a recent review, "development biologists have found dozens of proteins and genes that play a role in the growth of plants and animals, but how growing organs and organisms can sense their size and know when to stop, is still a mystery" [47]. The paradox is that it is well known what happens when many signaling pathways are interrupted, but we do not know what they do when they work properly.

In this work we have revisited the puzzle of size control for a system growing with a spatially homogeneous mitotic rate in terms

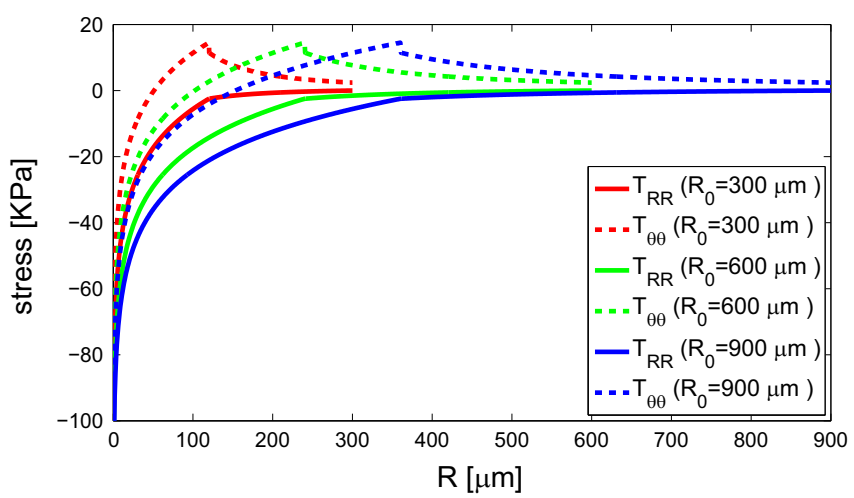

Fig. 6. Plot of the radial and circumferential stress components versus the radial coordinate $R$ at different domain sizes for a non-linear elastic material. Here duplication occurs only in the central region of the disk, bounded by $R_{i} ; \mu=40$ and $\gamma=1$ and $\delta=(1 / 0.9)^{2}$. Not surprisingly, the functions strongly resemble the results of the linear theory.

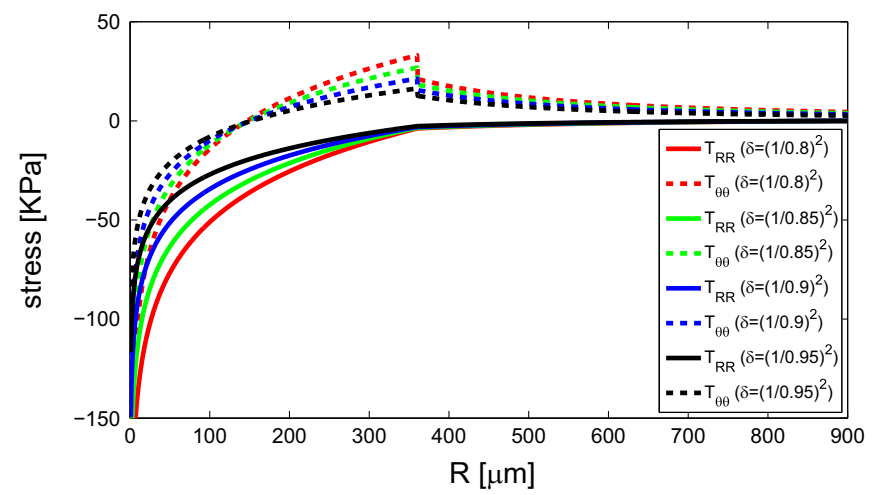

Fig. 7. Plot of the radial and circumferential stress components versus the radial coordinate $R$ at different values of the parameter $\delta$, corresponding to variable magnitude of the cell contractility. The other parameters are as in Fig. 6. of a precise physical and mathematical meaning: a (chemical or mechanical) field $f$ is acceptable signal if its spatial dependence according to the laws of physics is a function of the domain size $R_{0}$ times a self-similar solution in space:

$f(R, t)=f_{0}\left(R_{0}\right) f_{1}\left(R / R_{0}\right)$.

In this scenario, the function $f_{0}$ provides the absolute information of the domain size, while $f_{1}$ is constant for a given cell because of homogeneous growth. In other words, a physical field is an admissible "measure" if it is a function of the domain length times a self-similar solution of the space.

The application of this formal sieve to possible explanations of the morphogenetic process excludes that solute transport and degrada-tion without scaling or homogeneous stress can provide a global mechanism for the transmission of size information. Simple reac-tiondiffusion models or homogeneous passive stress are inadequate to transmit global information, as the corresponding fields do not behave as in (31). Conversely, reaction-diffusion of solutes where the characteristic length scales with the disc radius, is an admissible "measure" mechanism [17], although the diluting arguments that would confirm the scaling is still to be experimentally confirmed.

On a different vein, we have shown that the gradient of stress actively produced by cells circumferentially polarized satisfy a number of experimentally reported features: it is compressive, the pressure being larger in the center of the disc. Most important, tension can be an effective communication mechanism as its gradient is of the form (31).

The exposed mechanical theory can be seen as complementary to the large literature that demonstrates the crucial role of morphogens in controlling shape and size. In this respect, Hufnagel et al. [18] explore a cooperation of morphogens concentration and stress: the latter might support the former where the concentration of solutes is very small. Our morpho-mechanical model differs from their work in two points: the tension is actively produced by the cells, and the morphogen has possibly a permissive role, delimiting the activation area by its own concentration. The radial variation of inhomogeneous stress, actively produced by the cells in a region spanned by morphogen concentration, results in an effective signal for domain length. Moreover, the pressure in the active region grows with the domain size, as experimentally observed. We remark that the morphogenetic factors actually involved as well their dynamics are immaterial for the validity of our main results: an inhomogeneous active stress is a good candidate mechanism of size signaling.

Summarizing, the mathematical model illustrated above is able to account for all the (few) known features of morphogen and stress dynamics in the growing wing imaginal disc of the drosophila. By elementary physical mechanisms framed first in the linear and then in the finite elastic theory, we devise a local measure the disc size, namely the stress variation, that can serve as global communication mechanism about the organ size. The choice of the specific morpho-genetic system at hand has been dictated by the wide literature available on the subject; unfortunately quantitative measures of stress and strain, that are usual in continuum mechanics, are still missing and no further comparison can be carried out. However, all the qualitative known features are correctly reproduced: large compres-sion in the disc pouch, circumferential polarization of the cells and growth of the pressure with the wing size.

At our knowledge, this work represents the first attempt to design a mathematical model, based on first physical principles, to validate and possibly falsify mechanisms of size determination. We remark that only four parameters are required, all of them with precise physical meaning (two material moduli, the circumferen-tial active strain and the activation radius $R_{i}$ ), while other authors mention a number of adjustable parameters for their models [48]. The assumption of radial symmetry of a two-dimensional wing here adopted allows to determine an analytical solution of the mec- 


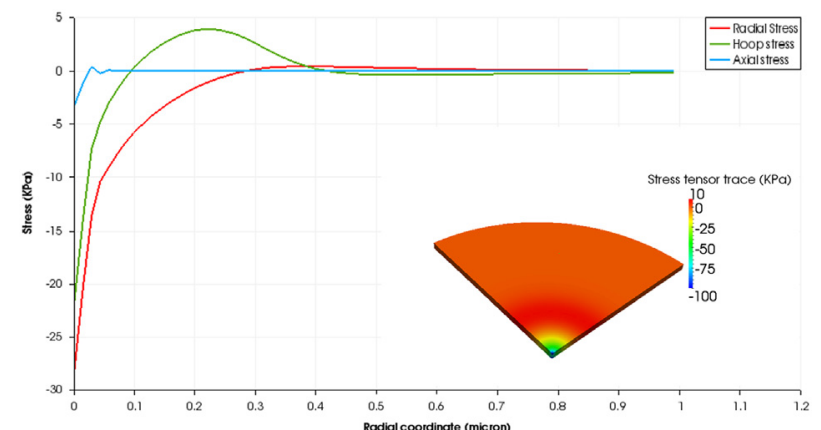

Fig. 8. Principal components of the stress tensor versus the radial coordinate, computed by a 3D non-linear finite element simulation. We considered a disk with an initial aspect ratio $H / R=0.02$, where $H$ is the thickness of the disk, and we assumed an incompressible non-linear behavior, setting $\mu=330 \mathrm{kPa}$, with a transversely isotropic active strain tensor, with a hoop contraction given by $a=0.8$. The inset depicts the simulated disk, whose colorbar represents the spatial distribution of the trace of the stress tensor. (For interpretation of the references to color in this figure caption, the reader is referred to the web version of this paper.)

hanical control on the self-similar growth pattern of the imaginal disc. The theory has been developed in a two-dimensional axial symmetric framework, suggested by the large scale ratio of the disc radius versus thickness. A posteriori, we are able to validate such an assumption on the basis of a full three dimensional non-linear finite elements numerical simulation. The three principal components of the stress versus the radial coordinate are plotted in Fig. 8. The axial stress is much smaller than the other components, thus corroborat-ing our two-dimensional assumption.

Finally, we feel that some of the ideas here exposed might be applied also to other morphogenetic systems, like embryos. The present approach is consistent with the emerging primary role of mechanotransduction in development [20], and pushes towards the development of novel experimental studies for confirming the key role of active stress for the global size control in morphoge-netic processes.

\section{Acknowledgments}

This work is partially funded by the "Start-up Packages and PhD Program" project, co-funded by Regione Lombardia through the "Fondo per lo sviluppo e la coesione 2007-2013", formerly FAS program.

\section{Appendix}

The displacement $u(R)$ in the case of diffusion-limited contractility takes the form

$u=A R^{\beta}-R, \quad R \in\left[0, R_{i}\right]$

$u=B R+\frac{C}{R}, \quad R \in\left[R_{i}, R_{0}\right]$,

where $\beta>1$. Since the radial stress must vanish at the outer boundary, it holds

$\frac{E}{1-\nu^{2}}\left(B-\frac{C}{R^{2}}\right)+\left.\frac{E \nu}{1-\nu^{2}}\left(B+\frac{C}{R^{2}}\right)\right|_{R=R_{0}}=0 \Rightarrow C=\frac{1+\nu}{1-\nu} B R_{0}^{2}$.

The continuity of the radial stress at the interface rewrites

$$
\begin{aligned}
\frac{E}{1-\nu^{2}}\left(A \beta R_{i}^{\beta-1}-1\right)+\frac{E \nu}{1-\nu^{2}} a^{2} A R_{i}^{\beta-1}= & \left.\frac{E B}{1-\nu^{2}} 1-\frac{1+\nu}{1-\nu}\left(\frac{R_{0}}{R_{i}}\right)^{2}\right) \\
& +\frac{E \nu B}{1-\nu^{2}}\left(1+\frac{1+\nu}{1-\nu}\left(\frac{R_{0}}{R_{i}}\right)^{2}\right),
\end{aligned}
$$

and we obtain

$$
A=\frac{(1+\nu)\left[\begin{array}{ll}
B & \left.1-\frac{R_{0}^{2}}{R_{i}^{2}}\right)+1
\end{array}\right]}{\left[\left(\beta+\nu a^{2}\right)\right] R_{i}^{\beta-1}} .
$$

To find the value of the constant $B$, we use the continuity of the displacement in $R=R_{i}$ :

$$
\left.\frac{(1+\nu)\left[\left(1-\frac{R_{0}^{2}}{R_{i}^{2}}\right) B+1\right] R_{i}^{\beta}}{\left(\beta+\nu a^{2}\right) R_{i}^{\beta-1}}-R_{i}=B \quad R_{i}-\frac{1+\nu}{1-\nu} \frac{R_{0}^{2}}{R_{i}}\right),
$$

that leads to

$$
B=\frac{R_{i}\left(\beta+\nu a^{2}-1-\nu\right)}{(1+\nu)\left(1-\frac{R_{0}^{2}}{R_{i}^{2}}\right) R_{i}+\left(\frac{1+\nu}{1-\nu} \frac{R_{0}^{2}}{R_{i}}-R_{i}\right)\left(\beta+\nu a^{2}\right)} .
$$

Therefore,

$$
\begin{aligned}
& u=A R^{\beta}-R \\
& T_{R R}=\frac{E}{1-\nu}\left(\left[\begin{array}{ll}
B & \left.1-\frac{R_{0}^{2}}{R_{i}^{2}}\right)+1
\end{array}\right]\left(\frac{R}{R_{i}}\right)^{\beta-1}-1\right) \\
& T_{\Theta \Theta}=\frac{E}{1-\nu}\left(\frac{\left[\begin{array}{ll}
B & \left.1-\frac{R_{0}^{2}}{R_{i}^{2}}\right)+1
\end{array}\right]\left(a^{2}+\nu \beta\right)}{\beta+\nu a^{2}}\left(\frac{R}{R_{i}}\right)^{\beta-1}-1\right)
\end{aligned}
$$

in $R \in\left[0, R_{i}\right]$ and

$$
\begin{aligned}
& \left.u=B \quad R+\frac{1+\nu}{1-\nu} \frac{R_{0}^{2}}{R}\right) \\
& \left.T_{R R}=B \frac{E}{1-\nu} \quad 1-\frac{R_{0}^{2}}{R^{2}}\right) \\
& \left.T_{\Theta \Theta}=B \frac{E}{1-\nu} 1+\frac{R_{0}^{2}}{R^{2}}\right),
\end{aligned}
$$

in $R \in\left[R_{i}, R_{0}\right]$, with $B$ calculated in (32).

\section{References}

[1] A.M. Turing, The chemical basis of morphogenesis, Proc. R. Lond. Biol. Sci. B 237 (1952) 37-72.

[2] L. Wolpert, Positional information and the spatial pattern of cellular differentiation, J. Theor. Biol. 25 (1969) 1-47.

[3] K.P. Landsberg, R. Farhadifar, J. Ranft, D. Umetsu, T.J. Widmann, T. Bittig, A. Said, F. Jülicher, C. Dahmann, Increased cell bond tension governs cell sorting at the Drosophila anteroposterior compartment boundary, Curr. Biol. 19 (2009) 1-6.

[4] X. Ma, H.E. Lynch, P.C. Scully, M.S. Hutson, Probing embryonic tissue mechanics with laser hole drilling, Phys. Biol. 6 (3) (2009) 036004.

[5] N. Serrano, P.H. O'Farrell, Limb morphogenesis: connections between patterning and growth, Curr. Biol. 7 (1997) R186-R195.

[6] M.C. Gibson, D.A. Lehman, G. Schubige, Lumenal transmission of decapentaplegic in Drosophila imaginal discs, Dev. Cell 3 (2002) 451-460.

[7] W.M. Gelbart, The decapentaplegic gene: a TGF-beta homologue controlling pattern formation in Drosophila, Development 107 (1989) 65-74.

[8] P.A. Lawrence, G. Struhl, Morphogens, compartments, and pattern: lessons from Drosophila? Cell 85 (1996) 951-961.

[9] D. Rogulja, K.D. Irvine, Regulation of cell proliferation by a Morphogen gradient, Cell 123 (2005) 449-461.

[10] J.B. Gurdon, P.Y. Bourillot, Morphogen gradient interpretation, Nature 413 (2001) 797-803.

[11] L. Wolpert, Positional information revisited, Development 107 (1989) 3-12.

[12] R. Burke, K. Basler, Dpp receptors are autonomously required for cell proliferation in the entire developing Drosophila wing, Development 122 (1996) 2261-2269.

[13] T. Lecuit, W.J. Brook, M. Ng, M. Calleja, H. Sun, S.M. Cohen, Two distinct mechanisms for long-range patterning by Decapentaplegic in the Drosophila wing, Nature 381 (1996) 387-393. 
[14] F.A. Spencer, F.M. Hoffmann, W.M. Gelbart, Decapentaplegic: a gene complex affecting morphogenesis in Drosophila melanogaster, Cell 28 (1982) 451-461.

[15] A. Kicheva, P. Pantazis, T. Bollenbach, Y. Kalaidzidis, T. Bittig, F. Jülicher M. Gonzalez-Gaitan, Kinetics of morphogen gradient formation, Science 315 (2007) 521-525.

[16] O. Wartlick, P. Mumcu, A. Kicheva, T. Bittig, C. Seum, F. Jülicher, M. GonzalezGaitan, Dynamics of Dpp signaling and proliferation control, Science 331 (2011) 1154-1159.

[17] O. Wartlick, P. Mumcu, F. Jülicher, M. Gonzalez-Gaitan, Understanding morphogenetic growth control - lessons from flies, Nat. Rev. Mol. Cell Biol. 12 (2011) 594-604.

[18] L. Hufnagel, A.A. Teleman, H. Rouault, S.M. Cohen, B.I. Shraiman, On the mechanism of wing size determination in fly development, Proc. Natl. Acad. Sci. U.S.A. 104 (2007) 3835-3840.

[19] D. Ben-Zvi, N. Barkai, Scaling of morphogen gradients by an expansionrepression integral feedback control, Proc. Natl. Acad. Sci. U.S.A. 107 (2010) 6924-6929.

[20] M.A. Wozniak, C.S. Chen, Mechanotransduction in development: a growing role for contractility, Nat. Rev. Mol. Cell Biol. 10 (2009) 34-43.

[21] L.A. Taber, Towards a unified theory for morphomechanics, Philos. Trans. R. Soc. A 13 (2009) 3555-3583.

[22] L.A. Taber, R. Perucchio, Modeling heart development, J. Elast. 61 (1-3) (2000) 165-197.

[23] A. Tosin, D. Ambrosi, L. Preziosi, Mechanics and chemotaxis in the morphogenesis of vascular networks, Bull. Math. Biol. 68 (7) (2006) 1819-1836.

[24] U. Nienhaus, T. Aegerter-Wilmsen, C.M. Aegerter, Determination of mechanical stress distribution in Drosophila wing disc using photoelasticity, Mech. Dev. 126 (2009) 942-949.

[25] T. Schluckl, C.M. Aegerter, Photo-elastic properties of the wing imaginal of Drosophila, Eur. Phys. J. (2010) 111-115.

[26] C.P. Heisenberg, Y. Bellaïche, Forces in tissue morphogenesis and patterning, Cell 153 (2013) 948-962.

[27] Y. Mao, A.L. Tournier, A. Hoppe, L. Kester, B.J. Thompson, N. Tapon, Differential proliferation rates generate patterns of mechanical tension that orient tissue growth, EMBO J. 32 (2013) 2790-2803.

[28] T. Schaffter, From genes to organisms: bioinformatics system models and software (Ph.D. thesis), Ecole Polytechnique Federale de Lausanne (EPFL), Switzerland, 2014

[29] P. McHale, W.J. Rappel, H. Levine, Embryonic pattern scaling achieved by oppositely directed morphogen gradients, Phys. Biol. 3 (2006) 107-120.

[30] G. Schwank, S.F. Yang, S. Restrepo, K. Basler, Comment on dynamics of Dpp signaling and proliferation control, Science 335 (6067) (2012) 401.

[31] C. Martin-Castellanos, B.A. Edgar, A characterization of the effects of Dpp signaling on cell growth and proliferation in the Drosophila wing, Development 129 (2002) 1003-1013.

[32] D. Nellen, R. Burke, G. Struhl, K. Basler, Direct and long-range action of a DPP morphogen gradient, Cell 85 (1996) 357-368

[33] C.M. Lo, H.B. Wang, M. Dembo, Y.L. Wang, Cell movement is guided by the rigidity of the substrate, Biophys. J. 79 (1) (2000) 144-152.
[34] M. Rauzi, P.F. Lenne, Cortical forces in cell shape changes and tissue morphogenesis, Curr. Top. Dev. Biol. 95 (2011) 93-144.

[35] Y. Mao, A.L. Tournier, P.A. Bates, J.E. Gale, N. Tapon, B.J. Thompson, Planar polarization of the atypical myosin Dachs orients cell division in Drosophila, Genes Dev. 25 (2011) 131-136.

[36] P. Nardinocchi, L. Teresi, On the active response of soft living tissues, J. Elast. 88 (1) (2007) 27-39.

[37] D. Breen, T. Widmann, L. Bai, F. Julicher, C. Dahmann, Epithelial cell reconstruction and visualization of the developing drosophila wing imaginal disc, in: IEEE Symposium on Biological Data Visualization (BioVis), 2012.

[38] G. Forgacs, R.A. Foty, Y. Shafrir, M.S. Steinberg, Viscoelastic properties of living embryonic tissues: a quantitative study, Biophys. J. 74 (1998) 2227-2234.

[39] M.L. Manning, R.A. Foty, M.S. Steinberg, E.M. Schoetz, Coaction of intercellular adhesion and cortical tension specifies tissue surface tension, Proc. Natl. Acad. Sci. 107 (2010) 12517-12522.

[40] M. Willecke, F. Hamaratoglu, L. Sansores-Garcia, C. Tao, G. Halder, Boundaries of Dachsous Cadherin activity modulate the Hippo signaling pathway to induce cell proliferation, Proc. Natl. Acad. Sci. 105 (39) (2008) 14897-14902.

[41] D. Ambrosi, S. Pezzuto, Active stress vs. active strain in mechanobiology: constitutive issues, J. Elast. 107 (2) (2012) 199-212.

[42] D. Ambrosi, G.A. Ateshian, E.M. Arruda, S.C. Cowin, J. Dumais, A. Goriely, K. Garikipati, Perspectives on biological growth and remodeling, J. Mech. Phys. Solids 59 (4) (2011) 863-883.

[43] A.S.G. Curtis, G.M. Seehar, The control of cell division by tension or diffusion, Nature 274 (1978) 52-53.

[44] EK. Rodriguez, A. Hoger, A.D. McCulloch, Stress-dependent finite growth in soft elastic tissues, J. Biomech. 27 (4) (1994) 455-467.

[45] P. Ciarletta, Buckling Instability in Growing Tumor Spheroids, Phys. Rev. Lett. 110 (15) (2013) 158102.

[46] D. Bigoni, D. Zaccaria, Loss of strong ellipticity in non-associative elastoplasticity, J. Mech. Phys. Solids 40 (6) (1992) 1313-1331.

[47] G. Vogel, How do organs know when they have reached the right size? Science 340 (6137) (2013) 1156-1157.

[48] T. Aegerter-Wilmsen, M.B. Heimlicher, A.C. Smith, P. Berbier de Ruille, R.S. Smith, C.M. Aegerter, K. Basler, Integrating force sensing and signaling pathways in a model for the regulation of wing imaginal disc, Development 139 (2012) 3221-3231.

[49] U. Nienhaus, T. Aegerter-Wilmsen, C.M. Aegerter, In-vivo imaging of the drosophila wing imaginal disc over time: novel insights on growth and boundary information, Plos One 7 (10) (2012) e47594.

[50] T. Aegerter-Wilmsen, C.M. Aegerter, E. Hafen, K. Basler, Model for the regulation of size in the wing imaginal disc of Drosophila, Mech. Dev. 124 (2007) 318-326.

[51] B.I. Shraiman, Mechanical feedback as a possible regulator of tissue growth, Proc. Natl. Acad. Sci. U.S.A. 102 (2005) 3318-3323.

[52] S. Swarup, E.M. Verheyen, Wnt/wingless signaling in Drosophila, Cold Spring Harb. Perspect. Biol. 4 (2012) a007930. 